\title{
The Mechanisms Underlying Changes in Broad Dimensions of Psychopathology During Cognitive Behavioral Therapy for Social Anxiety Disorder
}

\author{
Sei Ogawa ${ }^{\mathrm{a}, \mathrm{c}}$, Risa Imai ${ }^{\mathrm{a}}$, Masako Suzuki ${ }^{\mathrm{a}}$, Toshi A. Furukawa ${ }^{\mathrm{b}}$, Tatsuo Akechi ${ }^{\mathrm{a}}$
}

\begin{abstract}
Background: Social anxiety disorder (SAD) patients commonly have broad dimensions of psychopathology. This study investigated the relationship between a wide range of psychopathology and attention or cognitions during cognitive behavioral therapy (CBT) for SAD.

Methods: We treated 96 SAD patients with group CBT. Using multiple regression analysis, we examined the associations between the changes in broad dimensions of psychopathology and the changes in self-focused attention or maladaptive cognitions in the course of CBT.

Results: The reduction in self-focused attention was related to the decreases in somatization, obsessive-compulsive, interpersonal sensitivity, anxiety, phobic anxiety, and global severity index. The reduction in maladaptive cognitions was associated with decreases in interpersonal sensitivity, depression, and global severity index.

Conclusions: The present study suggests that changes in self-focused attention and maladaptive cognitions may predict broad dimensions of psychopathology changes in SAD patients over the course of CBT. For the purpose of improving a wide range of psychiatric symptoms with SAD patients in CBT, it may be useful to decrease self-focus attention and maladaptive cognitions.
\end{abstract}

Keywords: Social anxiety disorder; Cognitive behavioural therapy; Broad dimensions of psychopathology; Mechanism

\section{Introduction}

Social anxiety disorder (SAD) patients commonly have broad

Manuscript submitted October 17, 2017, accepted October 26, 2017

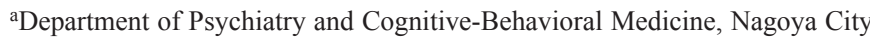
University Graduate School of Medical Sciences, Nagoya, Japan

${ }^{b}$ Department of Health Promotion and Human Behavior, Kyoto University Graduate School of Medicine/School of Public Health, Kyoto, Japan

${ }^{\mathrm{c} C}$ Corresponding Author: Sei Ogawa, Department of Psychiatry and CognitiveBehavioral Medicine, Nagoya City University Graduate School of Medical Sciences, Mizuho-cho, Mizuho-ku, Nagoya 467-8601, Japan.

Email: seiogawa1964@nifty.com

doi: https://doi.org/10.14740/jocmr3232w dimensions of psychopathology [1]. SAD with comorbid psychiatric disorders is thought to be severe condition [2].

The efficacy of cognitive behavioral therapy (CBT) for $\mathrm{SAD}$ has been well established [3]. Several studies reported that the effects of CBT may extend beyond targeted anxiety disorder symptoms to improvements in broad dimensions of psychopathology $[4,5]$.

Models of SAD suggest that heightened self-focused attention and maladaptive cognitions influence the development and maintenance of social anxiety. Self-focused attention is a shift in attention from external stimuli to detailed monitoring of the self in threatening social situations [6]. Reductions in self-focused attention and maladaptive cognitions may be associated with improvements in SAD symptoms after CBT [7, 8]. However, whether self-focused attention or maladaptive cognitions is related to a wide range of psychopathology during CBT has not been well studied.

This study examined the relationship between broad dimensions of psychopathology and attention or cognitions in the course of CBT for SAD.

\section{Materials and Methods}

\section{Participants}

One hundred ten Japanese SAD patients attended the group CBT program. Entry criteria were principal Axis I diagnosis of SAD according to the Diagnosis and Statistical Manual of Mental Disorders (fourth edition) [9], as evaluated by using the Structured Clinical Interview for the Diagnosis and Statistical Manual of Mental Disorders (fourth edition) [10]. Exclusion criteria for this study were current psychosis, bipolar disorder and substance use disorder. The patients provided their written informed consent after receiving a full explanation of the purpose and procedures of the study. The study was performed in accordance with the Declaration of Helsinki and the study's protocol was approved by the Ethics Committee of our institute.

\section{Treatments}

Our CBT program for SAD was originally based on the treatment manual developed by Andrews et al [11]. The program was composed of psychoeducation, behavioral experiments, 
Table 1. Predictors of SCL-90-R Subscales Changes $(n=96)$

\begin{tabular}{|c|c|c|c|c|c|c|c|c|c|c|}
\hline & $\triangle \mathrm{SOM}$ & $\Delta O-C$ & $\Delta \mathrm{I}-\mathrm{S}$ & $\triangle D E P$ & $\Delta \mathrm{ANX}$ & $\triangle \mathrm{HOS}$ & $\triangle \mathrm{PHOB}$ & APAR & $\Delta \mathrm{PSY}$ & $\Delta$ GSI \\
\hline$\triangle \mathrm{BFNE}$ & 0.17 & 0.22 & $0.34 *$ & $0.37 *$ & 0.28 & 0.13 & 0.07 & 0.25 & 0.30 & $0.31 *$ \\
\hline$\triangle$ FAQ self & $0.35^{*}$ & $0.47 * *$ & $0.36^{*}$ & 0.26 & $0.36^{*}$ & 0.07 & $0.38 *$ & 0.31 & 0.24 & $0.39 * *$ \\
\hline Adjusted $\mathrm{R}^{2}$ & 0.21 & 0.38 & 0.38 & 0.32 & 0.31 & 0.17 & 0.17 & 0.24 & 0.23 & 0.39 \\
\hline
\end{tabular}

Table shows the standardized beta coefficients. SCL-90-R: Symptom Checklist-90 Revised; BFNE: Brief Fear of Negative Evaluation Scale; FAQ: Focus of Attention Questionnaire; SOM: somatization; O-C: obsessive-compulsive; I-S: interpersonal sensitivity; DEP: depression; ANX: anxiety; HOS: hostility; PHOB: phobic anxiety; PAR: paranoid ideation; PSY: psychosis; GSI: global severity index. ${ }^{*} \mathrm{P}<0.005$. ${ }^{* *} \mathrm{P}<0.001$.

attention training, cognitive restructuring, and in vivo graded exposures. Two trained therapists conducted 2-h sessions once a week for 16 weeks.

\section{Measurements}

At pre- and post-treatment, all the patients were evaluated using following instruments.

The Symptom Checklist-90 Revised (SCL-90-R) is a universally used measure for general psychopathology. It is subdivided into 10 subscales of somatization, obsessive-compulsive, interpersonal sensitivity, depression, anxiety, hostility, phobic anxiety, paranoid ideation, psychoticism, and global severity index. Each item is scored between 0 (not at all) and 4 (extremely). The reliability and validity of the Japanese version have been demonstrated [12].

The Brief Fear of Negative Evaluation Scale (BFNE) is a 12-item self-reported questionnaire to assess fear of negative evaluation in social situations. The items are rated as on a five-point Likert type format, 0 (not at all) to 4 (extremely). Adequate reliability and validity have been shown [13].

The Focus of Attention Questionnaire (FAQ) is a 10-item scale composed of two five-item subscales: self-focused attention and external-focused attention [7]. We utilized selffocused attention subscale of FAQ because self-focused attention plays a causal role in SAD. The items are scored on a five-point Likert type, 0 (not at all) to 4 (extremely). Good reliability and validity have been reported [7].

\section{Statistical analysis}

The baseline and post-treatment data were used to calculate change scores for each variable, denoted by delta $(\Delta)$. For all outcome measures, the post-treatment score was subtracted from the baseline score. Therefore, positive $\Delta$ represented a change associated with worse broad dimensions of psychopathology.

All the data were analyzed using SPSS 18.0 for Windows [14]. First, we used an independent samples $t$-test or $\chi^{2}$ test to compare the baseline demographic and clinical data between the patients who finished the program and those who did not. Second, we performed multiple linear regression analysis using the variables about the changes in BFNE and self-focused attention subscale of FAQ as independent variables and the changes in the 10 subscales of SCL-90-R as dependent variables. All the statistical tests were two-tailed. Except for the multiple linear regression analysis, $\mathrm{P}<0.05$ was considered statistically significant. In the multiple linear regression analysis, we used the Bonferroni correction in order to counteract the problem of multiple comparison. We divided the original alpha value by the number of analyses on the dependent variable. The alpha value of less than 0.005 was considered statistically significant in the multiple linear regression analysis.

\section{Results}

Fourteen patients out of 110 who entered the treatment dropped out prematurely from the group CBT program. There was no statistically significant difference in the baseline characteristics between the subgroups.

In multiple regression analysis (Table 1), the reduction in self-focused attention subscale of FAQ was associated with the decreases in somatization, obsessive-compulsive, interpersonal sensitivity, anxiety, phobic anxiety, and global severity index. The reduction in BFNE was related to decreases in interpersonal sensitivity, depression, and global severity index.

\section{Conclusion}

The present study showed that the reductions in self-focused attention and maladaptive cognitions predicted broad dimensions of psychopathology reductions in SAD patients over the course of CBT.

A novel finding of our study is that the changes in self-focused attention may predict the changes in anxiety symptoms, whereas the changes in maladaptive cognitions may predict the changes in depression symptoms during CBT for SAD. For the purpose of improving anxiety symptoms with SAD patients in CBT, it may be useful to decrease self-focus attention. Moreover, it may be beneficial for SAD patients with comorbid depression symptoms to reduce maladaptive cognitions.

The present study has a number of limitations. First, this work was carried out as a single-arm and naturalistic study. Second, antidepressant and benzodiazepine medications were permitted during CBT. It is hard to consider dose effects of medications on this treatment program. Third, we used only self-reported questionnaires to assess dependent and independent variables.

\section{Acknowledgments}

SO was the principal investigator of this study. TAF supervised 
this work. All the authors participated in the clinical research.

\section{Conflict of Interest}

There is no conflict of interest.

\section{Funding}

This study was supported by a Grant-in-Aid for Scientific Research from the Ministry of Health, Labour and Welfare, Japan.

\section{References}

1. Kessler RC, Chiu WT, Demler O, Merikangas KR, Walters EE. Prevalence, severity, and comorbidity of 12-month DSM-IV disorders in the National Comorbidity Survey Replication. Arch Gen Psychiatry. 2005;62(6):617-627.

2. Schneier FR, Johnson J, Hornig CD, Liebowitz MR, Weissman MM. Social phobia. Comorbidity and morbidity in an epidemiologic sample. Arch Gen Psychiatry. 1992;49(4):282-288.

3. Acarturk C, Cuijpers P, van Straten A, de Graaf R. Psychological treatment of social anxiety disorder: a metaanalysis. Psychol Med. 2009;39(2):241-254.

4. Craske MG, Farchione TJ, Allen LB, Barrios V, Stoyanova M, Rose R. Cognitive behavioral therapy for panic disorder and comorbidity: more of the same or less of more? Behav Res Ther. 2007;45(6):1095-1109.

5. Tsao JC, Lewin MR, Craske MG. The effects of cognitive-behavior therapy for panic disorder on comorbid conditions. J Anxiety Disord. 1998;12(4):357-371.

6. Higa CK, Daleiden EL. Social anxiety and cognitive biases in non-referred children: The interaction of self-focused attention and threat interpretation biases. J Anxiety Disord. 2008;22(3):441-452.

7. Woody SR, Chambless DL, Glass CR. Self-focused attention in the treatment of social phobia. Behav Res Ther. 1997;35(2):117-129.

8. Heimberg RG, Liebowitz MR, Hope DA, Schneier FR, Holt CS, Welkowitz LA, Juster HR, et al. Cognitive behavioral group therapy vs phenelzine therapy for social phobia: 12-week outcome. Arch Gen Psychiatry. 1998;55(12):1133-1141.

9. American Psychiatric Association. Task Force on DSMIV. Diagnostic and statistical manual of mental disorders : DSM-IV. 4th ed. Washington, DC: American Psychiatric Association; 1994.

10. First MB. Structured clinical interview for DSM-IV axis I disorders: SCID - I: clinician version: administration booklet. Washington, D.C.: American Psychiatric Press; 1997.

11. Andrews G, Creamer M, Crino R, Hunt C, Lampe L, Page A. The treatment of anxiety disorders: clinician guides and patient manuals. 2nd ed. Cambridge, UK; New York, NY: Cambridge University Press; 2003.

12. Furukawa TA, Nakanishi M, Sakurai A, Suzuki A, Suzuki-Moor A, Hamanaka T. Effects of rthyl loflazepate in mood and neurosis-related disorders (ICD-10 JCM) ; Changes in SCL-90-R subscale scores. Rinsho Seisinigaku (Clinical Psychiatry). 1996;25:233-240.

13. Ishikawa R, Sasaki K, Fukui I. Standardization of Japanese version of FNE and SADS. Koudou Ryouhou Kenkyu (Jap J Behav Ther). 1992;18:10-17.

14. SPSS. SPSS for Windows (Version 18.0). 2009. 